\title{
Dsg2 Upregulation as a Rescue Mechanism in Pemphigus
}

\author{
Anna M. Sigmund, Letyfee S. Steinert, Desalegn T. Egu, Franziska C. Bayerbach, \\ Jens Waschke and Franziska Vielmuth*
}

Department I, Faculty of Medicine, Institute of Anatomy and Cell Biology, Ludwig-Maximilians-Universität, Munich, Germany

In pemphigus vulgaris (PV), autoantibodies directed against the desmosomal cadherin desmoglein (Dsg) 3 cause loss of intercellular adhesion. It is known that Dsg3 interactions are directly inhibited by autoantibody binding and that Dsg2 is upregulated in epidermis of PV patients. Here, we investigated whether heterophilic Dsg2-Dsg3 interactions occur and would modulate PV pathogenesis. Dsg2 was upregulated in PV patients' biopsies and in a human ex vivo pemphigus skin model. Immunoprecipitation and cell-free atomic

OPEN ACCESS

Edited by: Attila Mócsai,

Semmelweis University, Hungary

Reviewed by:

Takashi Hashimoto,

Osaka City University, Japan

Hong Wan,

Queen Mary University of London,

United Kingdom

*Correspondence:

Franziska Vielmuth

Franziska.Vielmuth@med.unimuenchen.de

Specialty section: This article was submitted to Autoimmune and Autoinflammatory Disorders, a section of the journal

Frontiers in Immunology

Received: 08 July 2020 Accepted: 28 September 2020 Published: 28 October 2020

Citation:

Sigmund AM, Steinert LS, Egu DT, Bayerbach FC, Waschke J and Vielmuth $F$ (2020) Dsg2

Upregulation as a Rescue Mechanism in Pemphigus.

Front. Immunol. 11:581370. doi: 10.3389/fimmu.2020.581370 force microscopy (AFM) experiments demonstrated heterophilic Dsg2-Dsg3 interactions. Similarly, in Dsg3-deficient keratinocytes with severely disturbed intercellular adhesion Dsg2 was upregulated in the desmosome containing fraction. AFM revealed that Dsg2Dsg3 heterophilic interactions showed binding frequency, strength, $\mathrm{Ca}^{2+}$-dependency and catch-bond behavior comparable to homophilic Dsg3-Dsg3 or homophilic Dsg2Dsg2 interactions. However, heterophilic Dsg2-Dsg3 interactions had a longer lifetime compared to homophilic Dsg2-Dsg2 interactions and PV autoantibody-induced direct inhibition was significantly less pronounced for heterophilic Dsg2-Dsg3 interactions compared to homophilic Dsg3 interactions. In contrast, a monoclonal anti-Dsg2 inhibitory antibody reduced heterophilic Dsg2-Dsg3 and homophilic Dsg2-Dsg2 binding to the same degree and further impaired intercellular adhesion in Dsg3-deficient keratinocytes. Taken together, the data demonstrate that Dsg2 undergoes heterophilic interactions with Dsg3, which may attenuate autoantibody-induced loss of keratinocyte adhesion in pemphigus.

Keywords: pemphigus, autoimmune blistering diseases, epidermis, keratinocyte, desmosome

\section{INTRODUCTION}

Desmosomes are highly regulated protein complexes mediating strong intercellular adhesion in tissues constantly exposed to high mechanical stress such as skin and heart $(1,2)$. They are composed of the desmosomal cadherins desmoglein (Dsg) 1-4 and desmocollin (Dsc) 1-3, which mediate intercellular adhesion in a $\mathrm{Ca}^{2+}$-dependent manner (3). Desmosomal cadherins are linked via plaque proteins such as plakoglobin (PG), plakophilins (Pkp), and desmoplakin (DP) to the intermediate filament cytoskeleton (4).

Desmosomal cadherins are known to interact with their counterparts in a homo- and heterophilic manner (5-8). For those interactions, an interaction mechanism comparable to the 
tryptophan swap model of classical cadherins is proposed. According to this model, a conserved tryptophan at position 2 binds in a hydrophobic pocket at the $\mathrm{EC} 1$ of the binding partner on the opposing cell membrane (trans-) or on the same cell (cisinteraction) (3). In the epidermis, desmosomal proteins show a differentiation and thus layer-specific expression pattern (9). For instance, Dsg1 and Dsg3 show a reversed distribution gradient with predominant expression of Dsg3 in the basal layers.

The importance of desmosomal proteins for tissue integrity is reflected in several desmosome-related diseases such as the genetic disorder arrhythmogenic cardiomyopathy or the bullous autoimmune disease pemphigus (10). The autoantibody profile of the latter correlates with the clinical phenotype of the disease (11). In pemphigus vulgaris (PV), autoantibodies mainly directed against Dsg1 and 3 cause suprabasal blistering of the epidermis and mucous membranes, whereas in pemphigus foliaceus (PF) autoantibodies directed against Dsg1 cause superficial blisters in the skin only. Accordingly, Dsg3 knock out (ko) mice show a mild PV like phenotype with mainly oral lesions and PV typical loss of cell-cell adhesion in suprabasal layers $(12,13)$. In contrast, Dsg1 deficiency resulted in lethal PF-like superficial skin blistering in mice (14).

According to our current knowledge, pemphigus autoantibodies directly inhibit Dsg3 interactions $(5,8)$ and induce dysregulation of several signaling pathways (15). Activation of p38 mitogen-activated protein (MAP) kinase (16-18), sarcoma associated $(\operatorname{Src})$ kinase $(19,20)$, extracellular signal-regulated kinase (ERK) $(21,22)$, and protein kinase C (2325) by autoantibodies contribute to Dsg1 and 3 internalization and uncoupling of keratins from the desmosome subsequently leading to loss of intercellular adhesion and blister formation in PV. Besides Dsg1 and 3, numerous other non-desmoglein autoantibodies found in PV targeting important proteins in physiology and cell adhesion, which may contribute to pemphigus pathogenesis (26). Nevertheless, the exact pathomechanisms in pemphigus are not fully elucidated.

It was reported that Dsg2 is upregulated in PV patients' lesions (27) and that Dsg2 and Dsg3 expression are interdependent in oral squamous cell carcinomas (28). Similarly, Dsg3-deficient keratinocytes showed a higher membrane localization of Dsg2 compared to wild type (wt) cells, and disturbed intercellular adhesion in Dsg3-depleted keratinocytes was impaired further after additional knock down of Dsg2 (29). Nevertheless, the biologic significance of Dsg2 upregulation for PV pathogenesis is unknown.

It has been shown that both Dsg2 and Dsg3 can undergo heterophilic binding with Dsc isoforms $(8,30)$. Further, Dsg3 binding events and unbinding forces were increased in keratindeficient keratinocytes as shown by cell-free atomic force microscopy (AFM) measurements (6), and Dsg3 binding was partially blocked by a monoclonal inhibitory anti-Dsg2 antibody. Based on these observations, we speculated that in situations of compromised Dsg3-mediated cell adhesion, e.g., keratin-deficiency or pemphigus, Dsg2 may be capable of compensating for loss of intercellular adhesion. Therefore, we here examined the biophysical properties of heterophilic
Dsg2-Dsg3 interactions and whether upregulation of Dsg2 affect pemphigus pathogenesis.

\section{MATERIALS AND METHODS}

For extended methods, please refer to the Supplementary Materials and Methods.

\section{Cell Culture and Establishment of Spontaneously Immortalized Mouse Keratinocytes}

The stable mouse epidermal keratinocytes (MEK) cell line was generated by spontaneously immortalization from Dsg3 ko and wt B6;129X1-Dsg $3^{\operatorname{tm} 1 S t a n} / \mathrm{J}$ mice as described before (Jackson Laboratory, Maine, US) (31). Briefly, epidermis was obtained from neonatal mice by incubation of the skin with Dispase II (Sigma-Aldrich, Munich, Germany) overnight. Epidermal cells were isolated after treatment for $1 \mathrm{~h}$ with Accutase (SigmaAldrich, Munich, Germany) and seeded in complete FAD media (0.05mM $\mathrm{CaCl}_{2}$, PAN Biotech, Aidenbach, Germany) on collagen I (rat tail; BD Bioscience, New Jersey, US) coated flasks and maintained at $35^{\circ} \mathrm{C}$ and $5 \% \mathrm{CO}_{2}$. After passaging for 10-15 times, keratinocytes immortalized spontaneously, while other epidermal cells die. For experiments, confluent cells were switched to high $\mathrm{Ca}^{2+}(1.2 \mathrm{mM})$ for $48 \mathrm{~h}$ to induce differentiation.

Human keratinocyte cells (HaCaT) (32) were maintained in Dulbecco's modified Eagle's medium (DMEM) (Life Technologies; Carlsbad; CA; USA) supplemented with $10 \%$ fetal calf serum (Biochrom, Berlin, Germany), 50 units $/ \mathrm{ml}$ penicillin (AppliChem, Darmstadt, Germany), $50 \mu \mathrm{g} / \mathrm{ml}$ streptomycin (AppliChem), and $1.8 \mathrm{mM} \mathrm{Ca}^{2+}$ at $37^{\circ} \mathrm{C}$ and $5 \% \mathrm{CO}_{2}$.

\section{Purification of Recombinant Dsg-Fc Constructs}

Purification of recombinant Dsg-Fc proteins was performed as described before (5). Briefly, extracellular domain-Fc constructs were expressed stably in Chinese hamster ovary $(\mathrm{CHO})$ cells, and recombinant proteins were isolated from supernatants by protein A agarose affinity chromatography (Life Technologies/Thermo Fisher Scientific, Waltham, USA).

\section{Atomic Force Microscopy}

Atomic force microscopy (AFM) utilizes a sharp tip on a flexible cantilever, which repetitively approaches and retrieves a probe. A laser beam is directed to the cantilever and its deflection, while the cantilever contacts the surface, is registered on a photodiode. During each approach-retract cycle, a force-distance curve is created by recording the deflection of the scanning tip. Analysis of force-distance curves allows to draw conclusions about the mechanical properties of the probe. By functionalizing the scanning tip and the probe with recombinant adhesion molecules, such as the extracellular domain of desmosomal 
cadherins, their respective interaction can be probed and the biophysical properties can be characterized $(33,34)$.

For AFM measurements, a NanoWizard 3 AFM (JPK Instruments, Berlin, Germany) mounted on an inverted optical microscope (Carl Zeiss, Jena, Germany) or a Nanowizard 4 AFM (JPK Instruments, Berlin, Germany) mounted on an inverted optical microscope (IX73 Olympus, Hamburg, Germany) were used in a cell-free setup described in detail before (6). Briefly, cantilever of silicon nitride MLCT AFM probes (Bruker, Mannheim, Germany) and silicon nitride mica sheets (SPI Supplies, West Chester, USA) were functionalized with corresponding Dsg-Fc constructs as illustrated before (35). A flexible heterobifunctional benzaldehyde polyethylenglycol (PEG) linker (BroadPharm, San Diego, US) was used to link proteins $(0.15 \mathrm{mg} / \mathrm{ml})$ to the respective surface. Measurements were done with the triangular D-tip with nominal spring constant of $0.03 \mathrm{~N} / \mathrm{m}$ and tip radius of $20 \mathrm{~nm}$ using Force Mapping mode. By evaluation of the resulting force-distance curves, adhesive properties of specific molecules can be analyzed (36). Beside the probability of an interaction to occur, referred to as binding frequency, the strength of the interaction, so called unbinding force, can be determined.

\section{Keratinocyte Dissociation Assay}

Dispase-based keratinocyte dissociation assay was performed as described before (37). Briefly confluent cells were switched to high $\mathrm{Ca}^{2+}$ for $48 \mathrm{~h}$, and monolayer was detached from culture well by Dispase II (Sigma Aldrich) supplemented with $1 \%$ collagenase I (Thermo Fisher Scientific). Afterwards, a defined shear stress was applied, and resulting fragments were counted. Number of fragments reversely correlates with intercellular adhesion.

\section{Immunoprecipitation}

Co-immunoprecipitation of HaCaT lysates (RIPA buffer, $50 \mathrm{mM}$ Tris- $\mathrm{HCl}, \mathrm{pH} 8 ; 150 \mathrm{mM} \mathrm{NaCl} ; 0.1 \%$ SDS; 1\% NP-40; $1 \mathrm{mM}$ EDTA) using $1.5 \mu \mathrm{g}$ of aDsg3 pAb (Biozol, Eching, Germany), aDsg2 pAb (Abbexa, Cambridge, UK) or a polyclonal rabbit ctrIgG was performed as described previously (29). Briefly, $1 \mathrm{mg}$ of precleared lysates were incubated together with the respective antibody for $3 \mathrm{~h}$ at $4^{\circ} \mathrm{C}$ on a rotating incubator. To precipitate antibody bound proteins, $30 \mu \mathrm{l}$ of $\mathrm{A} / \mathrm{G}$ beads (Santa Cruz Biotechnology, Dallas, TX, USA) were added for an overnight incubation at $4^{\circ} \mathrm{C}$ and subsequent centrifugation. Beads were washed with RIPA buffer, and precipitated proteins were analyzed by Western blotting. For co-immunoprecipitation after crosslinking of surface proteins, cells were treated with the membrane impermeable crosslinker DTSSP (3,3'-dithiobis [sulfosuccinimidylpropionate], ThermoScientific, Waltham, USA) according to the manufacturer's instructions.

\section{Western Blot}

Cell lysis and triton extraction were performed as described elsewhere in detail (37) and in Supplementary Materials and
Methods. For detection of cell surface proteins, a biotinylation assay was performed using a protocol modified from Vielmuth et al. (38). In brief, confluent cells were incubated with membrane impermeable EZ-Link Sulfo-NHS-Biotin (ThermoScientific, Waltham, USA) and lysed in RIPA buffer (50 mM Tris- $\mathrm{HCl}, \mathrm{pH}$ 8; $150 \mathrm{mM} \mathrm{NaCl} ; 0.1 \%$ SDS; 1\% NP-40; $1 \mathrm{mM}$ EDTA) and centrifuged. Biotinylated surface proteins from pellet and supernatant were precipitated with NeutrAvidin HighCapacity agarose beads (Thermo Scientific, Waltham, USA) and analyzed by Western blot.

SDS-PAGE (SDS-polyacrylamide gel electrophoresis) followed by Western blot analyses was conducted as shown previously (37).

\section{Ex Vivo Pemphigus Skin Model}

Human ex vivo pemphigus model was performed with cadavers of the human body donor program without history of skin diseases from the Institute of Anatomy and Cell Biology, Ludwig-Maximilians-Universität Munich, Germany as described before (17). Briefly, skin biopsis were divided into $1 \times 1 \mathrm{~cm}$ pieces and intradermal injection of PV-IgG or ctr-IgG were conducted. Specimen were incubated for $24 \mathrm{~h}$ in DMEM at $37^{\circ} \mathrm{C}$ at $5 \% \mathrm{CO}_{2}$ and subsequently subjected to a defined mechanical stress (six times scraping with constant pressure using a soft rubber). Finally, skin pieces were embedded in TissueTec (Leica Biosystems, Nussloch, Germany) for cryo cutting using a cryostat microtome (HM 500 OM, Microm International GmbH, Walldorf, Germany).

\section{Immunostaining}

Staining of ex vivo and patient samples was performed after standard protocol as described before (17). Images were taken using a Leica SP5 confocal microscope with a 63x NA 1.4 PL APO objective controlled by LAS AF software (Leica, Mannheim, Germany).

\section{Ethic Statement}

The studies involving human participants were reviewed and approved by Ethics Committee of the University of Würzburg (Az159/06), the Ludwig-Maximillians-Universität of Munich, the University of Budapest (Az48825-5/2019/EÜIG), the University of Marburg (Az20/14), and the University of Lübeck (Az12-178). The patients/participants provided their written informed consent to participate in this study.

\section{Data Processing and Statistics}

Analysis of AFM force-distance curves was done with JPK data processing software and peak fit analysis and Bells equation was done in Origin Pro 2016, 93G (Northampton, MA). For processing and creation of figures, Adobe Photoshop CS4 (Adobe, Dublin, Ireland) was used. For graphs and statistics Prism 5 (GraphPad Software, San Diego, US) was used. For quantification of 
immunostainings, ImageJ-Software was used to determine the mean of raw intensities of epidermal cells divided by the area.

\section{RESULTS}

\section{Pemphigus Autoantibodies Induce Upregulation of Dsg2 in Human Epidermis}

Dsg3 ko mice suffer from pemphigus-like lesions (12, 13). Primary keratinocytes derived from these mice show a highly disturbed intercellular adhesion. Interestingly, Dsg2, which is almost absent in healthy, adult epidermis, was localized along cell borders in these Dsg3 ko keratinocytes $(29,37)$. Thus, we wondered whether Dsg2 upregulation could serve as a compensatory mechanism in pemphigus. To investigate whether Dsg2 upregulation is present in pemphigus lesions, we used a human ex vivo pemphigus skin model. Healthy human skin from body donors was injected with either control IgG or PV1-IgG of pemphigus patient 1 (Table 1), incubated for $24 \mathrm{~h}$, and subjected to mechanical stress. Samples injected with control IgG revealed normal expression patterns of Dsg1 and 3 with higher Dsg1 expression in the superficial epidermal layers and Dsg3 expression predominantly in the basal and suprabasal layers (Figure 1A). Dsg2 was almost absent along cell membranes as described before but showed diffuse cytoplasmic staining (Figure 1B) $(37,39)$. In contrast, PV1-IgG injection resulted in reduced and fragmented Dsg1 and Dsg3 staining, further referred to as Dsg depletion as described before (40) (Figure 1A). In contrast, Dsg2 staining in PV1-IgG injected samples was increased, which was particularly pronounced in keratinocytes surrounding the blisters where autoantibodies were bound predominantly (Figures 1B, arrows, C). Staining against human IgG confirmed binding of autoantibodies in PV-IgG injected samples only. Specificity of staining was shown by secondary antibody controls (Figure S1A). Similar results were obtained with injection of PV2-IgG (data not shown). To address specificity of this upregulation, we stained ex vivo samples for the desmosomal proteins Dsc1 and Dsc3. Both of them are described to be targets of autoantibodies in IgA pemphigus or $\mathrm{PV}$, respectively $(11,41)$. Nevertheless, upregulation upon PVIgG injection seemed to be specific for Dsg2 because neither Dsc1 nor Dsc3 staining in PV4-IgG injected samples was

TABLE 1 | Dsg titers of pemphigus patient samples.

\begin{tabular}{lcccc}
\hline Patient & Dsg1 [U/ml] & Dsg3 [U/ml] & Clinical phenotype & Sample \\
\hline 1 & 168,1 & 174,64 & PV & IgG \\
2 & 760 & 4711 & PV & IgG \\
3 & 1,5 & 154 & PV & lgG \\
4 & n.d. & n.d. & PV & lgG \\
5 & 22 & 310 & PV & skin \\
6 & - & 243 & PV & skin \\
7 & 19,7 & 725 & PV & skin \\
8 & 34 & 3038 & PV & skin \\
\hline
\end{tabular}

n.d., Not defined; purified lgG samples of pemphigus patients (IgG), perilesional skin biopsies of pemphigus patients (skin). Cut-offs were at $20 \mathrm{U} / \mathrm{ml}$. enhanced (Figures S1B, C). Similar results were obtained with injection of PV1- and PV2-IgG (data not shown).

Next, we analyzed Dsg2 expression in perilesional pemphigus patient samples (Table 1). Dsg1 staining of patient 5 was fragmented in the basal layers but not significantly reduced (Figure 1D and Figure S2A), but the patient showed Dsg3 depletion depicted by deffuse cytoplasmic staining (Figure 1E and Figure S2B). Interestingly, 2 out of 4 patient samples showed increased localization of Dsg2 at the membrane of epidermal keratinocytes, which was absent in all samples of healthy epidermis (Figures 1F, G). Binding of autoantibodies was tested by staining against human IgG and secondary antibody controls showed specificity of the staining (Figures S2C, D). Taken together, these data suggest that pemphigus autoantibodies induce Dsg2 upregulation.

\section{Dsg2 and Dsg3 Interact Heterophilically}

Ex vivo and patient data suggest that Dsg2 could contribute to pemphigus pathogenesis as a compensatory mechanism. Former studies showed that Dsg3 interacts homo- and heterophilically with some desmosomal cadherins $(5,6,8,42)$. Thus, we here characterized whether Dsg3 would also undergo heterophilic interaction with Dsg2.

To investigate the heterophilic interaction of Dsg2 and Dsg3 in keratinocytes, we first performed immunoprecipitation using an anti-Dsg3 or anti-Dsg2 antibody in human keratinocytes (HaCaT). Indeed, Dsg2 co-immunoprecipitated with Dsg3 and vice versa showing the occurrence of heterophilic Dsg2-Dsg3 interactions in human keratinocytes (Figure 2A). We further applied the membrane-impermeable crosslinker DTSSP to assure extracellular interactions of Dsg2 and Dsg3. Indeed, IP after crosslinking confirmed interaction of Dsg2 and Dsg3 (Figure S3A).

Next, we characterized heterophilic Dsg2-Dsg3 interactions using cell-free AFM measurements. AFM measurements using tips and mica sheets coated with recombinant Fc-constructs of Dsg2- and Dsg3- extracellular domains revealed similar binding frequency for Dsg3-Dsg3 and Dsg2-Dsg2 homo- and Dsg2-Dsg3 heterophilic interactions (Figure 2B). Similarly, the strength of single molecule interaction, further referred as unbinding force (UF) did not differ between homophilic Dsg2-Dsg2 and Dsg3Dsg3 interactions and heterophilic Dsg2-Dsg3 interactions (Figure 2C).

Similar to classical cadherins, homophilic interactions of desmosomal cadherins require $\mathrm{Ca}^{2+}(5,43-45)$. However, it remains unclear whether heterophilic Dsg2-Dsg3 interactions reveal a similar $\mathrm{Ca}^{2+}$ dependency like homophilic interactions of Dsg2 and Dsg3. Thus, we depleted $\mathrm{Ca}^{2+}$ by application of the $\mathrm{Ca}^{2+}$-chelator EGTA in cell-free AFM measurements. EGTA significantly lowered the binding frequency of homophilic Dsg3Dsg3 and of the heterophilic Dsg2-Dsg3 interactions to a similar extent (Figure 2D) indicating that heterophilic interactions are also $\mathrm{Ca}^{2+}$-dependent.

To further characterize the biophysical properties of heterophilic Dsg2-Dsg3 interactions, we determined their bond lifetime. AFM measurements with different pulling speeds from 1 to $20 \mu \mathrm{m} / \mathrm{s}$ revealed increasing UF with 


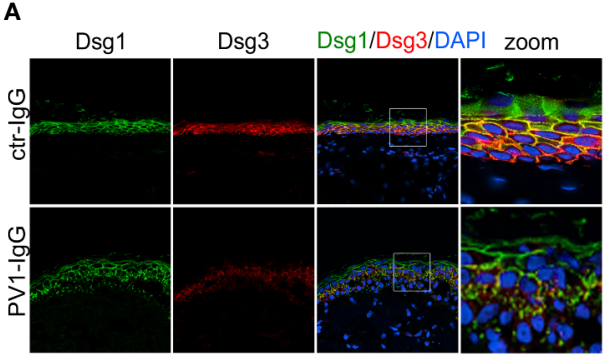

C

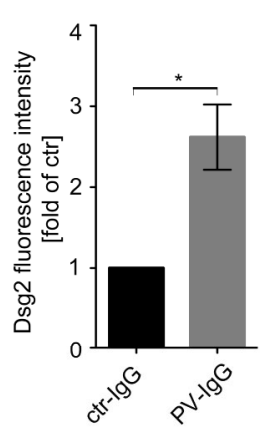

$\mathbf{F}$

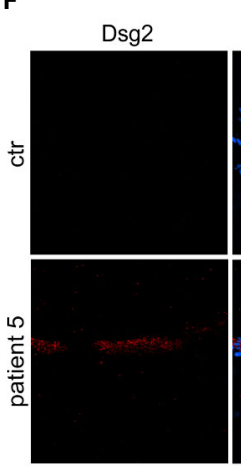

D
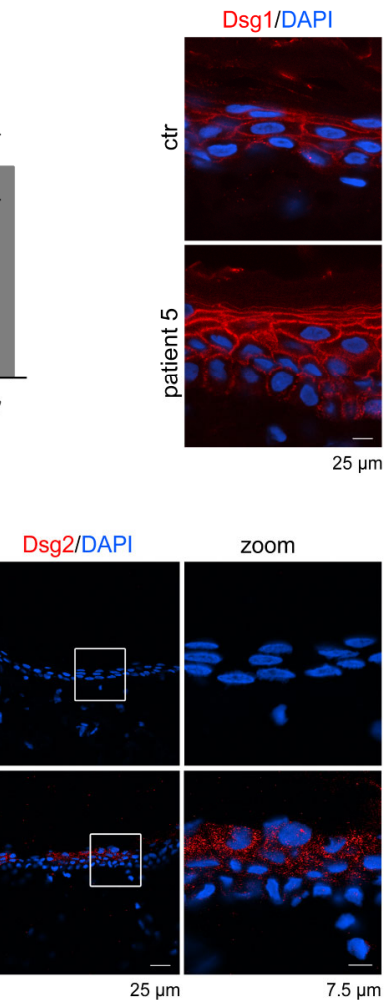

B

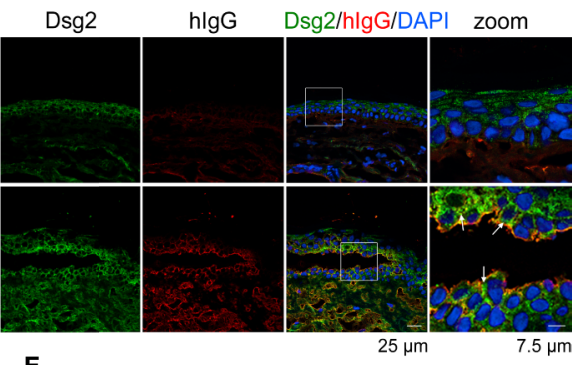

E

Dsg3/DAPI

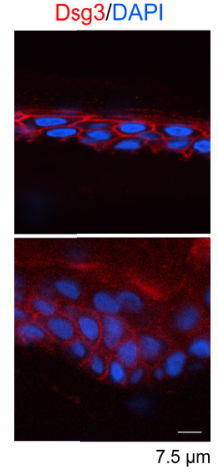

G

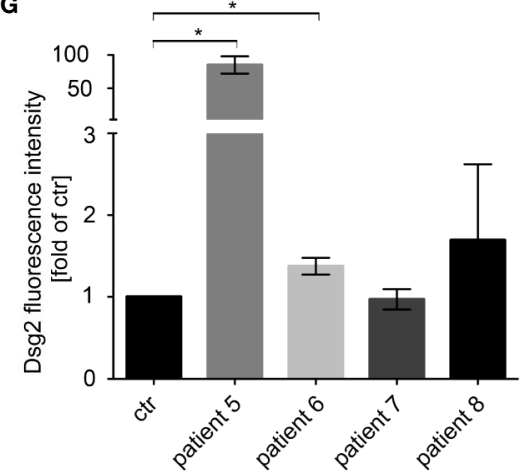

FIGURE 1 | Pemphigus autoantibodies induce Dsg2 upregulation. Immunostaining against Dsg1, Dsg3 (A) and Dsg2 and human (h) IgG (B) in human ex vivo skin after injection with PV1-lgG or control (ctr) IgG from healthy humans. Dsg2 is upregulated in PV-lgG treated samples, especially along blisters (arrows).

(C) Quantification of Dsg2 staining of perilesional cells of PV-lgG injected samples or control epidermal cells $(n=3$ with PV1- and PV2-lgG). Pemphigus patient samples were stained against Dsg1 (D), Dsg3 (E) and Dsg2 (F). Untreated ex vivo skin served as a control. Images show 1 of 2 Dsg2 positive samples out of 4 patients (patient 5-8). (G) Quantification of Dsg2 staining of patient 5-8 revealing upregulation of Dsg2 in pemphigus patients. Nuclei were stained with DAPI. Columns indicate mean value normalized to $\mathrm{ctr} \pm \mathrm{SEM}$, ${ }^{\star} \mathrm{P}<0.05$; student $\mathrm{t}$-test to $\mathrm{ctr}$. desmoglein (Dsg), pemphigus vulgaris (PV).

increasing pulling speeds and loading rates as depicted in a dot plot of UF against logarithmic loading rates at various pulling speeds (Figure 2E). This indicates that Dsg2-Dsg3 interactions behave like catch-bonds, which is in line with former studies on Dsg interactions $(6,34)$. By plotting the extreme fitted values of UFs against loading rates and fitting them in a modified Bells equation, we determined a bond lifetime of $0.22 \mathrm{~s}$ for heterophilic interactions $(46,47)$. This was almost doubled compared to lifetimes of homophilic Dsg2 interactions which were 0.12 s (Figure 2F).

Taken together, these data show that heterophilic Dsg2-Dsg3 interactions behave similar to the homophilic Dsg3 interactions in terms of binding frequency, strength, and $\mathrm{Ca}^{2+}$-dependency. In contrast, increased bond lifetime of the heterophilic Dsg2Dsg3 interactions may stabilize keratinocyte adhesion under conditions when Dsg3-mediated cell adhesion is disturbed by pemphigus autoantibodies.

\section{Murine Dsg3 ko Keratinocytes Show Dsg2 Upregulation}

To further analyze this potential compensatory Dsg2 upregulation mechanism we generated a stable murine Dsg3 ko keratinocyte cell line (MEK) from neonatal mice. 
A

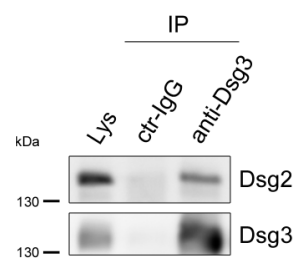

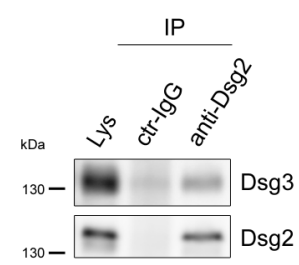

B

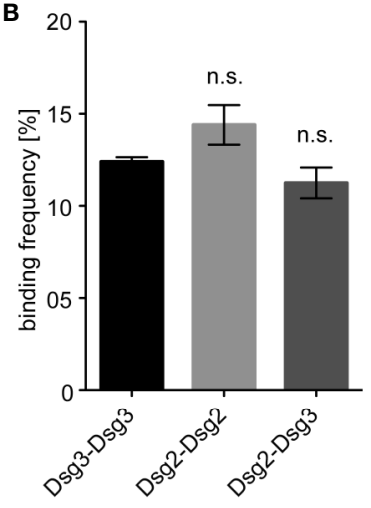

C

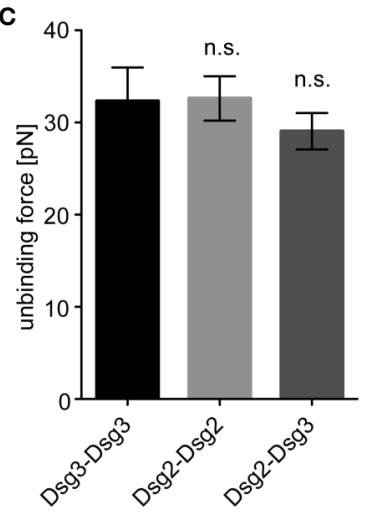

E
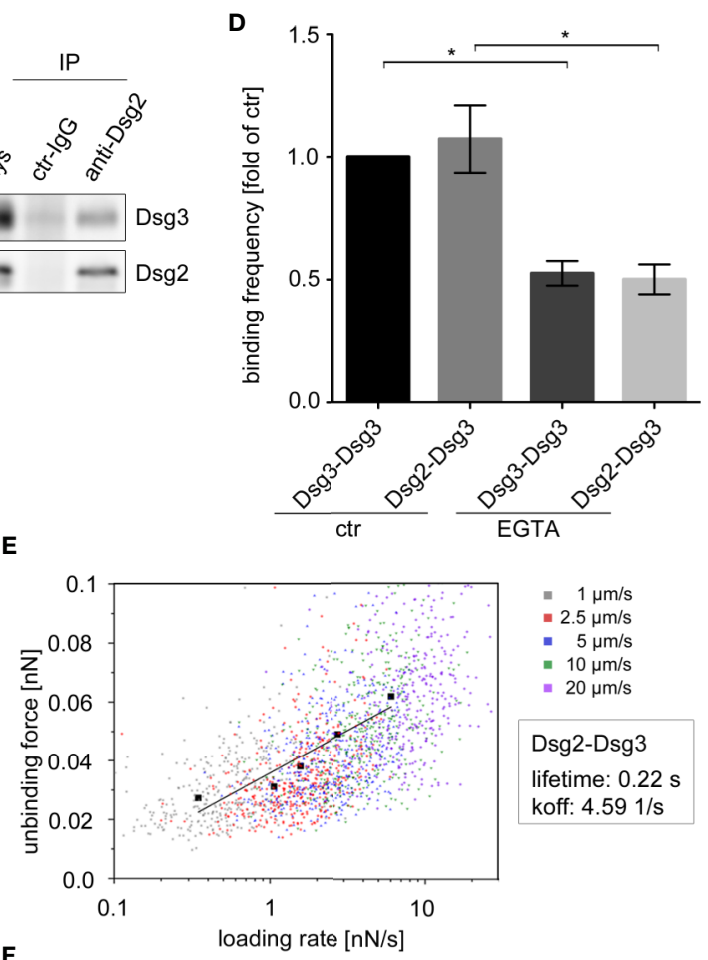

- $1 \mu \mathrm{m} / \mathrm{s}$

- $2.5 \mu \mathrm{m} / \mathrm{s}$

- $\begin{array}{r}5 \mu \mathrm{m} / \mathrm{s} \\ \text { - } 10 \mu \mathrm{m} / \mathrm{s}\end{array}$

- $20 \mu \mathrm{m} / \mathrm{s}$

Dsg2-Dsg3

lifetime: $0.22 \mathrm{~s}$

koff: $4.591 / \mathrm{s}$

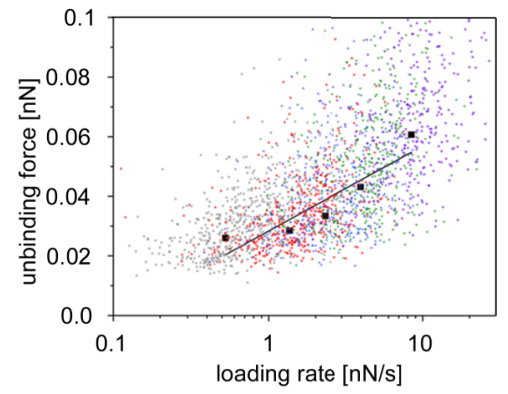

- $1 \mu \mathrm{m} / \mathrm{s}$

- $2.5 \mu \mathrm{m} / \mathrm{s}$

- $5 \mu \mathrm{m} / \mathrm{s}$

- $10 \mu \mathrm{m} / \mathrm{s}$

Dsg2-Dsg2

lifetime: $0.12 \mathrm{~s}$

koff: $8.321 / \mathrm{s}$

FIGURE 2 | Dsg2 and Dsg3 interact heterophilically. (A) IP in human keratinocytes (HaCaT) with polyclonal anti-Dsg3 and anti-Dsg2 antibodies, or rabbit control (ctr) IgG showing heterophilic interactions of Dsg2 and Dsg3. Pure lysates (Lys) were loaded as a control. Representative of $n=4$. Binding frequencies (B) and unbinding forces (C) of cell-free AFM measurements probing homophilic Dsg3-Dsg3, Dsg2-Dsg2 and heterophilic Dsg2-Dsg3 interactions. Binding frequencies and unbinding forces are comparable throughout all interaction types. Columns indicate mean value \pm SEM ( $n=5$, each 2,500 force-distance curves). (D) Binding frequencies of cell-free AFM measurements probing Dsg3-Dsg3 and Dsg2-Dsg3 interactions with and without incubation of 5 mM EGTA for $1 \mathrm{~h}$. Columns indicate mean value normalized to $\mathrm{ctr} \pm \mathrm{SEM}$, *P < 0.05; One-way ANOVA with Bonferroni correction ( $\mathrm{n}=3$, each 2,500 force-distance curves). Dot plots for Dsg2-Dsg3 (E) and Dsg2Dsg2 (F) interactions of unbinding forces (UF) against logarithmic loading rates at various pulling speeds. Black graphs depict the extreme fitted values of UFs plotted against loading rates and fitted in a modified bells equation. Modified bells equation resulted in a bond lifetime of $0.22 \mathrm{~s}$ for heterophilic Dsg2-Dsg3 and of $0.12 \mathrm{~s}$ for homophilic Dsg2-Dsg2 interactions ( $n \geq 3$, each 2,500 (E) or 3,600 (F) force-distance curves). EGTA, ethylene glycol tetraacetic acid.

Dispase-based keratinocyte dissociation assay showed a highly disturbed intercellular adhesion with 10 times more fragments in the Dsg3 ko MEKs compared to cells derived from wt littermates (Figure 3A).

Western blot analysis of whole cell lysates, triton soluble, and desmosome-containing triton insoluble fraction showed that expression of Dsg2 was also elevated in Dsg3 ko cells compared to wt cells, in line with the observation that pemphigus autoantibodies induce upregulation of Dsg2 in human epidermis (Figure 3B). Levels of E-Cad were unchanged in the corresponding fraction. Protein levels in the desmosomal fraction of Dsg3 ko lysates of Dsc3, cytokeratin 14, and the plaque proteins PG and DP were decreased, whereas Dsc1 levels were increased compared to wt cells (Figure 3B). Additionally cell surface biotinylation in Dsg3-deficient MEKs confirmed increased Dsg2 localization at the plasma membrane compared to wt cells (Figure 3C). Detection of E-Cad served as a loading control. Of note, Dsg2 seemed to be located in a fraction, which was in contrast to Dsg3, PG, and E-Cad not soluble in RIPA buffer. These results imply that lack of Dsg3 resulted in an 

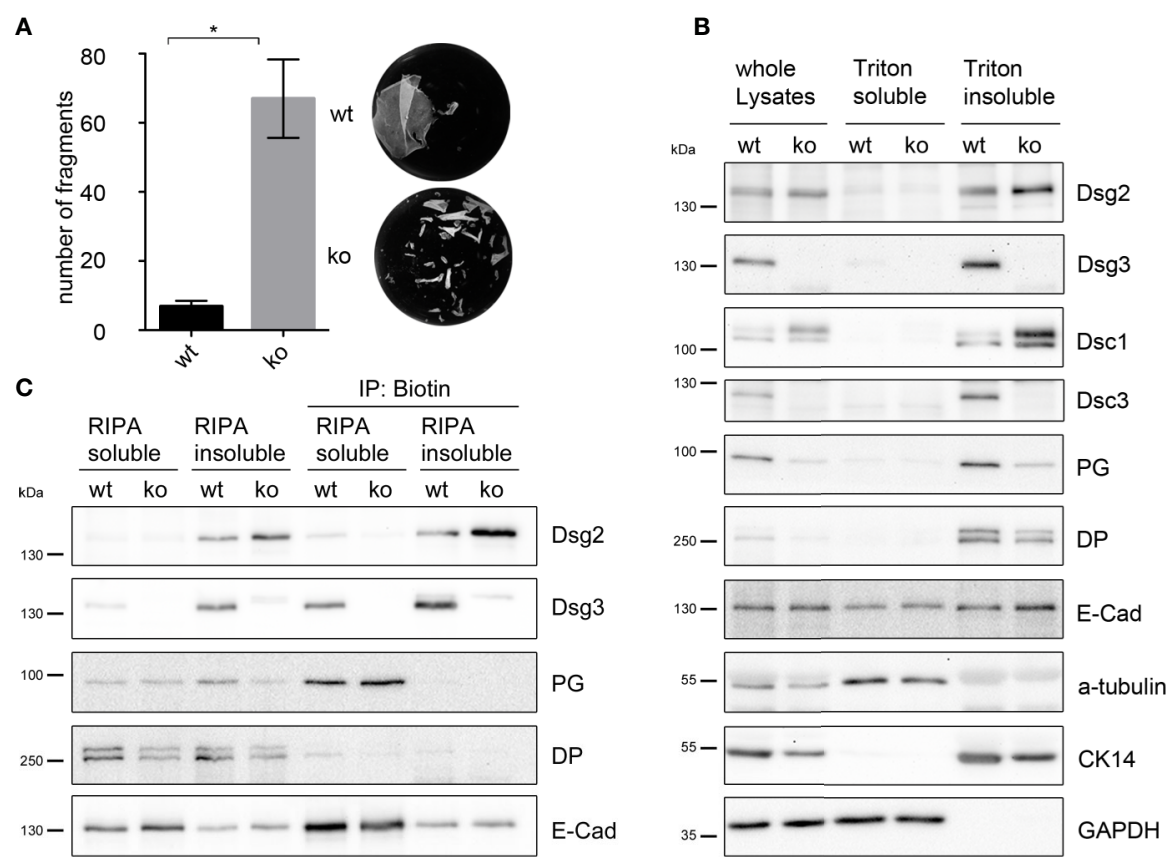

FIGURE 3 | Dsg2 is upregulated in murine Dsg3 ko keratinocytes. (A) Dispase-based keratinocyte dissociation assay of stable Dsg3 wt and ko keratinocytes showing compromised intercellular adhesion in cells lacking Dsg3. Columns indicate mean value $\pm \mathrm{SEM}$, ${ }^{*} P<0.05$; student $\mathrm{t}$-test $(\mathrm{n}=4)$. (B) Representative Western blot of Dsg3 wt and ko whole cell lysates, triton soluble and triton insoluble, desmosome containing fraction. Dsg3 ko cells show upregulation of Dsg2 and Dsc1 whereas Dsc3, cytokeratin 14 and the plaque proteins PG and DP were decreased compared to wt cells. E-Cad and GAPDH served as loading controls ( $\mathrm{n}=$ 4). (C) Representative Western blot of a biotinylation assay. RIPA buffer soluble and insoluble biotinylated membrane proteins were immunoprecipitated using biotin coated beads demonstrating Dsg2 upregulation at the cell membrane of Dsg3-deficient keratinocytes. E-cad served as loading control ( $n=4)$. DP, desmoplakin; CK, cytokeratin; Dsc, desmocollin; PG, plakoglobin; GAPDH, Glyceraldehyde 3-phosphate dehydrogenase.

upregulation of Dsg2 in desmosomes, which may contribute to rescue at least partly from disrupted cell cohesion.

\section{Heterophilic Dsg2-Dsg3 Interactions are Less Susceptible to Pemphigus Autoantibodies Than Homophilic Dsg3-Dsg3 Interactions}

Pemphigus autoantibodies directed against Dsg3 cause direct inhibition of homophilic and heterophilic Dsg3 interactions (5, $8,38)$. Thus, we wondered how pathogenic Dsg3 antibodies affect heterophilic Dsg2-Dsg3 interactions.

Therefore, we analyzed the heterophilic Dsg2-Dsg3 interactions by cell-free AFM measurements after treatment with AK23, a pathogenic anti-Dsg3 antibody, derived from a pemphigus mouse model (48). AK23 significantly reduced the binding frequency of homophilic Dsg3-Dsg3 interactions compared to untreated control (Figure 4A). In contrast, inhibition of heterophilic Dsg2-Dsg3 binding was less pronounced and AK23-treated heterophilic interactions showed a significant higher binding frequency compared to AK23-treated homophilic interactions. Moreover, AFM measurements also showed that Dsg2-Dsg3 interactions were less susceptible to PV3-IgG treatment than Dsg3-Dsg3 interactions (Figure 4B). In contrast to the pathogenic Dsg3-targeting autoantibodies, a monoclonal inhibitory anti-Dsg2 antibody inhibited both homophilic Dsg2-Dsg2 and heterophilic Dsg2-Dsg3 interactions to the same extent (Figure S3B).

Finally, we tested whether Dsg2 upregulation would protect to some extent against autoantibody-induced loss of cell cohesion. Therefore, we further analyzed the effect of AK23 on intercellular adhesion of murine keratinocytes in dissociation assays. AK23 increased the number of fragments in wt but not in Dsg3 ko MEKs (Figure 4C and Figure S3C). In contrast, treatment of Dsg3 ko cells with the inhibitory anti-Dsg2 antibody led to a drastic increase of fragments, implying that upregulated Dsg2 can partially compensate for deficiency of Dsg3 and thereby ameliorate loss of intracellular adhesion (Figure 4D).

\section{DISCUSSION}

Our data show an upregulation of Dsg2 in a human ex vivo pemphigus skin model and in pemphigus patient epidermis. Upregulation of Dsg2 allowed heterophilic interactions of Dsg2 with Dsg3. Because biophysical properties of heterophilic 
A

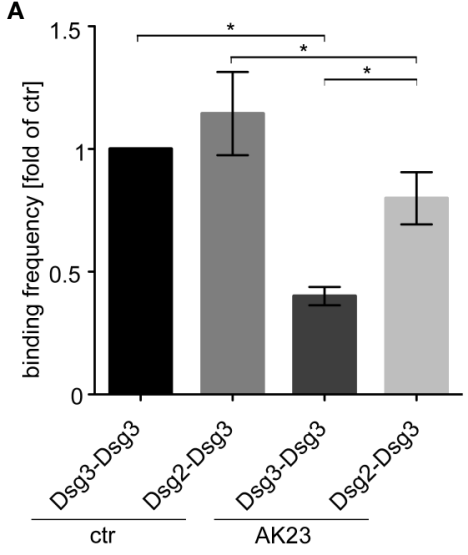

C

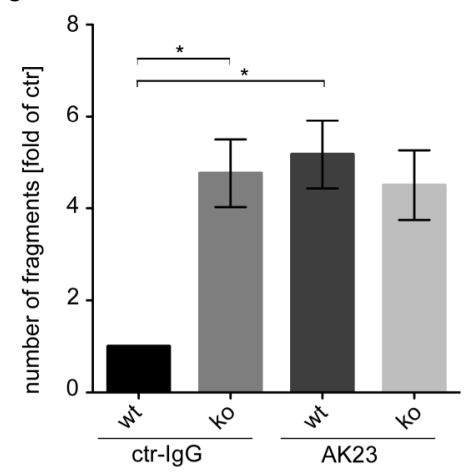

B

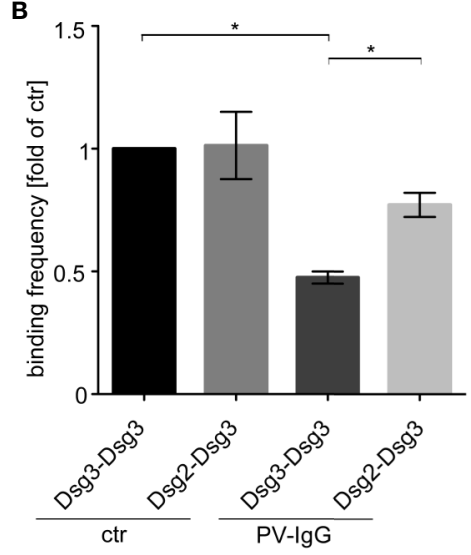

D

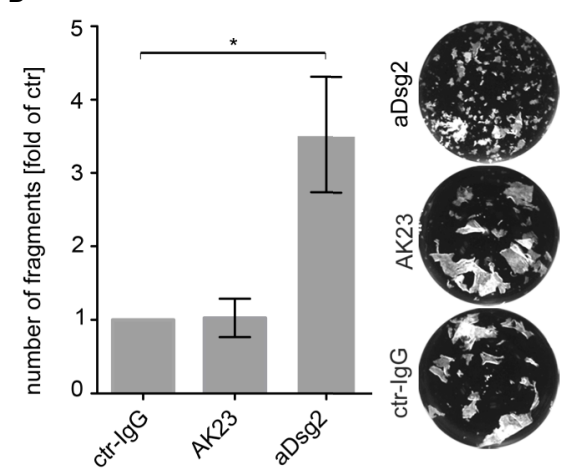

FIGURE 4 | Heterophilic Dsg2-Dsg3 interactions were less susceptible to autoantibody-induced direct inhibition of Dsg interaction. Binding frequencies of cell-free AFM measurements probing Dsg3-Dsg3 and Dsg2-Dsg3 interaction pairs with and without (ctr) incubation of either a monoclonal anti-Dsg3 antibody (AK23) (A) or PV3-IgG (B) for $1 \mathrm{~h}$ ( $\mathrm{n} \geq 5$, each 2500 force-distance curves). Heterophilic Dsg2-Dsg3 interactions are less susceptible against direct inhibition by pathogenic Dsg3 antibodies than homophilic Dsg3 interactions. (C) Dispase-based keratinocyte dissociation assay of Dsg3 wt and ko keratinocytes after treatment with control (ctr) IgG or AK23 for $24 \mathrm{~h}$ showing loss of intercellular adhesion after AK23 in wt cells only $(\mathrm{n}=3)$. (D) Keratinocyte dissociation assay of Dsg3 ko keratinocytes after treatment with ctr-lgG, AK23 or an inhibitory anti-Dsg2 antibody (aDsg2) for 24 h revealing drastic effect of aDsg2 antibody in cells lacking Dsg3 ( $\mathrm{n}=4$ ). Columns indicate mean value normalized to $\mathrm{ctr} \pm \mathrm{SEM},{ }^{*} \mathrm{P}<0.05$; One-way ANOVA with Bonferroni correction.

interactions of Dsg2-Dsg3 are in part different from homophilic Dsg interactions, the data indicate that heterophilic interactions may be advantageous. First, these heterophilic interactions show a higher bond lifetime than homophilic Dsg2-Dsg2 interactions. More important, they were less susceptible to direct inhibition by pathogenic pemphigus antibodies than homophilic Dsg3-Dsg3 interactions. Taken together, these data argue for a compensatory mechanism in pemphigus based on heterophilic Dsg2Dsg3 interactions.

\section{Biological Impact of Homo- and Heterophilic Dsg3 Interactions}

Desmosomal cadherins were reported to undergo homophilic and heterophilic interactions $(8,34,42,49)$. Data obtained by bead aggregation assays and surface plasmon resonance using the first extracellular domain of desmoglein isoforms indicated predominant occurrence of heterophilic interactions (8, 42). Nevertheless, in the study of Harrison et al., crystal structure analyses demonstrated homodimers of Dsg2 if high concentrations of complete molecules were applied which may reflect the situation in densely packed desmosomes (42). Using AFM, heterophilic interactions of Dsg2 with Dsc2 and Dsg2 with EGFR and Dsg1 with Dsc3 (7, 30, 50, 51), and homophilic interactions for all desmogleins were detected $(6,34)$. Biochemical cross-linking indicated that in keratinocytes homophilic interactions of Dsg isoforms are predominant (49). Collectively, these data indicate that Dsg can undergo both homophilic and heterophilic interactions. Possible differences may be caused by the techniques and model systems applied in which the mechanical load on the molecules differs. This was reported to be crucial for the function of several parts of cell-cell-adhesion structures (1, 52, 53). Further, distinct interactions could be preferred in dependency on the biological circumstances, differentiation of the cells and cellular behavior. Therefore, for this study, we combined biochemical methods and AFM analyses to study whether Dsg3 can undergo interactions with Dsg2. By co-immunoprecipitation of Dsg2 with Dsg3, we confirmed heterophilic interactions in human keratinocytes. 
However, IP data do not exclude the possibility of an interaction of Dsg2 and Dsg3 by cytoplasmic proteins. Thus, we further used cell-free AFM and detected both homophilic interactions of Dsg2 and Dsg3 and heterophilic interactions of Dsg2 with Dsg3, which is in accordance to previous studies $(6,38,54)$. Taken together, the data presented strongly argue for a direct interaction of Dsg2 and Dsg3 via their extracellular domains.

Both interaction types, i.e. homo- and heterophilic, were $\mathrm{Ca}^{2+}$ dependent and behaved like catch-bonds, which was described to be characteristic for cadherin-type adhesion $(34,55,56)$. Catchbonds are characterized by binding strength which increases with the load applied on the interaction (57). Interestingly, forces and binding probability were comparable for all interactions detected, whereas bond lifetime for heterophilic interactions was prolonged in comparison to homophilic interactions of Dsg2. Similar results were reported for the homophilic interactions of Dsg1 in comparison to the heterophilic interactions of Dsg1 to Dsc3 (7). Thus, one can speculate that homo- and heterophilic interactions may fulfill different biological functions and heterophilic interactions may be beneficial under conditions where intercellular adhesion is challenged such as in pemphigus.

\section{Dsg2 Is Upregulated When Homophilic Interactions of Dsg3 are Disturbed and may Protect Against PV-IgG-Induced Loss of Cell Adhesion}

Dsg2, except in hair follicles, is almost absent in healthy adult human epidermis $(37,36)$. In contrast, Dsg2 was reported to be upregulated in perilesional skin of pemphigus patients (27). Our data confirm upregulation of Dsg2 in pemphigus patient skin. Further, Dsg2 upregulation was present in a human ex vivo pemphigus model at membranes of cells bordering blisters suggesting that PV-IgGinduced loss of adhesion was causative for this phenomenon. This observation is in line with the hypothesis that perturbed Dsg3 adhesion caused upregulation of Dsg2 and both homophilic Dsg2Dsg2 and heterophilic Dsg2-Dsg3 interactions may compensate for loss of Dsg3 function in pemphigus. This supports previous data in cultured murine keratinocytes lacking Dsg3 in which Dsg2 was reported to partly compensate for loss of intercellular adhesion $(29,37)$. Interestingly, comparable to our ex vivo model, immunostaining of Dsg3-deficient keratinocytes revealed an increase of Dsg2 at cell membranes (37). Here, we further generated a stable Dsg3-deficient cell line, which showed disturbed cell adhesion and upregulation of Dsg2 in the insoluble, desmosome-containing fraction supporting the observations revealed by immunostaining of ex vivo and pemphigus patient epidermis. Taken together, these data suggest that Dsg3 function and Dsg2 expression may be interdependent.

Keratinocytes are capable of inducing rescue mechanisms to protect against PV-IgG-induced loss of intercellular adhesion. For instance, a cAMP increase was reported after PV-IgG treatment in human keratinocytes - a mechanism that is effective to prevent loss of intercellular adhesion when augmented by cAMP increasing mediators (58). Similarly,
Dsg2 upregulation could also serve as a cellular rescue mechanism in PV. This is in line with a study showing that mice after forced expression of Dsg2 in superficial epidermal layers were protected against PF-IgG-induced blister formation (59).

Therefore, it is conceivable that Dsg2 upregulation may also protect against loss of Dsg3 adhesion in response to antibody binding. It is known that Dsg3 interactions are disturbed by direct inhibition caused by autoantibodies $(5,8)$. Interestingly, heterophilic interactions between Dsg2 and Dsg3 were less susceptible to inhibition of trans-interaction in response to both the pathogenic Dsg3 antibody AK23 and PV-IgG. Importantly, a specific inhibitory anti-Dsg2 antibody further impaired loss of intercellular adhesion in Dsg3 ko keratinocytes, supporting the idea of a compensatory mechanism.

Taken together, the data support the hypothesis of an autoantibody-induced rescue mechanism based on Dsg2 upregulation and Dsg2-Dsg3 heterophilic interactions to ameliorate loss of intercellular adhesion in pemphigus.

\section{DATA AVAILABILITY STATEMENT}

The raw data supporting the conclusions of this article will be made available on request by the authors, without undue reservation.

\section{ETHICS STATEMENT}

The studies involving human participants were reviewed and approved by Ethics Committee of the University of Würzburg (Az159/06), the Ludwig-Maximillians-Universität of Munich, the University of Budapest (Az48825-5/2019/EÜIG), the University of Marburg (Az20/14), University of Lübeck (Az12-178). The patients/participants provided their written informed consent to participate in this study. The animal study was reviewed and approved by the Government of Upper Bavaria, (Az. 55.22532.Vet_02-14-139).

\section{AUTHOR CONTRIBUTIONS}

AS and FV conducted the experiments, acquired the data, and analyzed the data. AS, FV, and DE developed the methodology. FB and LS conducted the experiments. AS, FV, and JW designed the research studies. AS, FV, and JW wrote the manuscript. All authors contributed to the article and approved the submitted version.

\section{FUNDING}

This work was supported by DFG FOR 2497 TP5 to JW and TP6 to FV and Else-Kröner-Fresenius-Stiftung 2016_AW157 to FV and JW. 


\section{ACKNOWLEDGMENTS}

The authors thank Nadine Albrecht, Sabine Mühlsimer and Martina Hitzenbichler for excellent technical assistance; Matthias Goebeler (Department for Dermatology, Venereology and Allergology, University hospital Würzburg), Miklós Sárdy (Department of Dermatology, Derma-oncology and Venerology, University Budapest); Michael Hertl (Department for Dermatology and Allergology, University hospital Marburg) and Enno Schmidt (Department of Dermatology, University of

\section{REFERENCES}

1. Price AJ, Cost AL, Ungewiss H, Waschke J, Dunn AR, Grashoff C. Mechanical loading of desmosomes depends on the magnitude and orientation of external stress. Nat Commun (2018) 9(1):5284. doi: 10.1038/s41467-018-07523-0

2. Berika M, Garrod D. Desmosomal adhesion in vivo. Cell Commun Adhes (2014) 21(1):65-75. doi: 10.3109/15419061.2013.876018

3. Owen GR, Stokes DL. Exploring the Nature of Desmosomal Cadherin Associations in 3D. Dermatol Res Pract (2010) 2010:930401. doi: 10.1155/ 2010/930401

4. Kowalczyk AP, Green KJ. Structure, function, and regulation of desmosomes. Prog Mol Biol Transl Sci (2013) 116:95-118. doi: 10.1016/B978-0-12-3943118.00005-4

5. Heupel WM, Zillikens D, Drenckhahn D, Waschke J. Pemphigus vulgaris IgG directly inhibit desmoglein 3-mediated transinteraction. J Immunol (2008) 181(3):1825-34. doi: 10.4049/jimmunol.181.3.1825

6. Vielmuth F, Wanuske MT, Radeva MY, Hiermaier M, Kugelmann D, Walter E, et al. Keratins Regulate the Adhesive Properties of Desmosomal Cadherins through Signaling. J Invest Dermatol (2018) 138(1):121-31. doi: 10.1016/ j.jid.2017.08.033

7. Spindler V, Heupel WM, Efthymiadis A, Schmidt E, Eming R, Rankl C, et al. Desmocollin 3-mediated binding is crucial for keratinocyte cohesion and is impaired in pemphigus. J Biol Chem (2009) 284(44):30556-64. doi: 10.1074/ jbc.M109.024810

8. Ishii K, Yoshida K, Stanley JR, Yamagami J, Amagai M, Ishiko A. Pemphigus vulgaris and foliaceus IgG autoantibodies directly block heterophilic transinteraction between desmoglein and desmocollin. J Invest Dermatol (2020) 140(10):1919-26.e7. doi: 10.1016/j.jid.2020.02.010

9. Waschke J. The desmosome and pemphigus. Histochem Cell Biol (2008) 130 (1):21-54. doi: 10.1007/s00418-008-0420-0

10. Schmidt E, Kasperkiewicz M, Joly P. Pemphigus. Lancet (2019) 394 (10201):882-94. doi: 10.1016/S0140-6736(19)31778-7

11. Spindler V, Waschke J. Pemphigus-A Disease of Desmosome Dysfunction Caused by Multiple Mechanisms. Front Immunol (2018) 9:136. doi: 10.3389/ fimmu.2018.00136

12. Koch PJ, Mahoney MG, Ishikawa H, Pulkkinen L, Uitto J, Shultz L, et al. Targeted disruption of the pemphigus vulgaris antigen (desmoglein 3 ) gene in mice causes loss of keratinocyte cell adhesion with a phenotype similar to pemphigus vulgaris. J Cell Biol (1997) 137(5):1091-102. doi: 10.1083/ jcb.137.5.1091

13. Rotzer V, Hartlieb E, Winkler J, Walter E, Schlipp A, Sardy M, et al. Desmoglein 3-Dependent Signaling Regulates Keratinocyte Migration and Wound Healing. J Invest Dermatol (2015) 136(1):301-10. doi: 10.1038/ JID.2015.380

14. Kugelmann D, Radeva MY, Spindler V, Waschke J. Desmoglein 1 Deficiency Causes Lethal Skin Blistering. J Invest Dermatol (2019) 139 (7):1596-1599 e2. doi: 10.1016/j.jid.2019.01.002

15. Spindler V, Eming R, Schmidt E, Amagai M, Grando S, Jonkman MF, et al. Mechanisms Causing Loss of Keratinocyte Cohesion in Pemphigus. J Invest Dermatol (2018) 138(1):32-7. doi: 10.1016/j.jid.2017.06.022

16. Berkowitz P, Hu P, Warren S, Liu Z, Diaz LA, Rubenstein DS. p38MAPK inhibition prevents disease in pemphigus vulgaris mice. Proc Natl Acad Sci U.S.A. (2006) 103(34):12855-60. doi: 10.1073/pnas.0602973103
Lübeck) for providing patient material and Eva Hartlieb for helpful discussions.

\section{SUPPLEMENTARY MATERIAL}

The Supplementary Material for this article can be found online at: https://www.frontiersin.org/articles/10.3389/fimmu.2020. 581370/full\#supplementary-material

17. Egu DT, Walter E, Spindler V, Waschke J. Inhibition of p38MAPK signalling prevents epidermal blistering and alterations of desmosome structure induced by pemphigus autoantibodies in human epidermis. Br J Dermatol (2017) 177 (6):1612-8. doi: 10.1111/bjd.15721

18. Jolly PS, Berkowitz P, Bektas M, Lee HE, Chua M, Diaz LA, et al. p38MAPK signaling and desmoglein-3 internalization are linked events in pemphigus acantholysis. J Biol Chem (2010) 285(12):8936-41. doi: 10.1074/ jbc.M109.087999

19. Kugelmann D, Rotzer V, Walter E, Egu DT, Fuchs MT, Vielmuth. F, et al. Role of Src and Cortactin in Pemphigus Skin Blistering. Front Immunol (2019) 10:626. doi: 10.3389/fimmu.2019.00626

20. Chernyavsky AI, Arredondo J, Kitajima Y, Sato-Nagai M, Grando. SA. Desmoglein versus non-desmoglein signaling in pemphigus acantholysis: characterization of novel signaling pathways downstream of pemphigus vulgaris antigens. J Biol Chem (2007) 282(18):13804-12. doi: 10.1074/ jbc.M611365200

21. Walter E, Vielmuth F, Rotkopf L, Sardy M, Horvath ON, Goebeler. M, et al. Different signaling patterns contribute to loss of keratinocyte cohesion dependent on autoantibody profile in pemphigus. Sci Rep (2017) 7(1):3579. doi: 10.1038/s41598-017-03697-7

22. Egu DT, Kugelmann D, Waschke J. Role of PKC and ERK Signaling in Epidermal Blistering and Desmosome Regulation in Pemphigus. Front Immunol (2019) 10:2883. doi: 10.3389/fimmu.2019.02883

23. Osada K, Seishima M, Kitajima Y. Pemphigus IgG activates and translocates protein kinase $\mathrm{C}$ from the cytosol to the particulate/cytoskeleton fractions in human keratinocytes. J Invest Dermatol (1997) 108(4):482-7. doi: 10.1111/ 1523-1747.ep12289726

24. Cirillo N, Lanza A, Prime SS. Induction of hyper-adhesion attenuates autoimmune-induced keratinocyte cell-cell detachment and processing of adhesion molecules via mechanisms that involve PKC. Exp Cell Res (2010) 316 (4):580-92. doi: 10.1016/j.yexcr.2009.10.005

25. Spindler V, Endlich A, Hartlieb E, Vielmuth F, Schmidt E, Waschke J. The extent of desmoglein 3 depletion in pemphigus vulgaris is dependent on $\mathrm{Ca}(2$ $+)$-induced differentiation: a role in suprabasal epidermal skin splitting? Am J Pathol (2011) 179(4):1905-16. doi: 10.1016/j.ajpath.2011.06.043

26. Amber KT, Valdebran M, Grando SA. Non-Desmoglein Antibodies in Patients With Pemphigus Vulgaris. Front Immunol (2018) 9:1190. doi: 10.3389/fimmu.2018.01190

27. Iwatsuki K, Han GW, Fukuti R, Ohtsuka M, Kikuchi S, Akiba H, et al. Internalization of constitutive desmogleins with the subsequent induction of desmoglein 2 in pemphigus lesions. Br J Dermatol (1999) 140(1):35-43. doi: 10.1046/j.1365-2133.1999.02604.x

28. Teh MT, Parkinson EK, Thurlow JK, Liu F, Fortune F, Wan H. A molecular study of desmosomes identifies a desmoglein isoform switch in head and neck squamous cell carcinoma. J Oral Pathol Med (2011) 40(1):67-76. doi: 10.1111/ j.1600-0714.2010.00951.x

29. Hartlieb E, Rotzer V, Radeva M, Spindler V, Waschke J. Desmoglein 2 compensates for desmoglein 3 but does not control cell adhesion via regulation of p38 mitogen-activated protein kinase in keratinocytes. J Biol Chem (2014) 289(24):17043-53. doi: 10.1074/jbc.M113.489336

30. Shafraz O, Rubsam M, Stahley SN, Caldara AL, Kowalczyk AP, Niessen CM, et al. E-cadherin binds to desmoglein to facilitate desmosome assembly. Elife (2018) 7:e37629. doi: 10.7554/eLife.37629 
31. Kroger C, Loschke F, Schwarz N, Windoffer R, Leube RE, Magin TM. Keratins control intercellular adhesion involving PKC-alpha-mediated desmoplakin phosphorylation. J Cell Biol (2013) 201(5):681-92. doi: 10.1083/jcb.201208162

32. Boukamp P, Petrussevska RT, Breitkreutz D, Hornung J, Markham A, Fusenig NE. Normal keratinization in a spontaneously immortalized aneuploid human keratinocyte cell line. J Cell Biol (1988) 106(3):761-71. doi: 10.1083/jcb.106.3.761

33. Zlatanova J, Lindsay SM, Leuba SH. Single molecule force spectroscopy in biology using the atomic force microscope. Prog Biophys Mol Biol (2000) 74(1-2):37-61. doi: 10.1016/S0079-6107(00)00014-6

34. Vielmuth F, Spindler V, Waschke J. Atomic Force Microscopy Provides New Mechanistic Insights into the Pathogenesis of Pemphigus. Front Immunol (2018) 9:485. doi: 10.3389/fimmu.2018.00485

35. Ebner A, Wildling L, Kamruzzahan AS, Rankl C, Wruss J, Hahn CD, et al. A new, simple method for linking of antibodies to atomic force microscopy tips. Bioconjug Chem (2007) 18(4):1176-84. doi: 10.1021/bc070030s

36. Carvalho FA, Santos NC. Atomic force microscopy-based force spectroscopybiological and biomedical applications. IUBMB Life (2012) 64(6):465-72. doi: 10.1002/iub.1037

37. Hartlieb E, Kempf B, Partilla M, Vigh B, Spindler V, Waschke J. Desmoglein 2 is less important than desmoglein 3 for keratinocyte cohesion. PloS One (2013) 8(1):e53739. doi: 10.1371/journal.pone.0053739

38. Vielmuth F, Waschke J, Spindler V. Loss of Desmoglein Binding Is Not Sufficient for Keratinocyte Dissociation in Pemphigus. J Invest Dermatol (2015) 135(12):3068-77. doi: 10.1038/jid.2015.324

39. Mahoney MG, Hu Y, Brennan D, Bazzi H, Christiano AM, Wahl JK 3rd. Delineation of diversified desmoglein distribution in stratified squamous epithelia: implications in diseases. Exp Dermatol (2006) 15(2):101-9. doi: 10.1111/j.1600-0625.2006.00391.x

40. Saito M, Stahley SN, Caughman CY, Mao X, Tucker DK, Payne AS, et al. Signaling dependent and independent mechanisms in pemphigus vulgaris blister formation. PloS One (2012) 7(12):e50696. doi: 10.1371/ journal.pone.0050696

41. Hashimoto T, Kiyokawa C, Mori O, Miyasato M, Chidgey MA, Garrod DR, et al. Human desmocollin 1 (Dscl) is an autoantigen for the subcorneal pustular dermatosis type of IgA pemphigus. J Invest Dermatol (1997) 109 (2):127-31. doi: 10.1111/1523-1747.ep12319025

42. Harrison OJ, Brasch J, Lasso G, Katsamba PS, Ahlsen G, Honig B, et al. Structural basis of adhesive binding by desmocollins and desmogleins. Proc Natl Acad Sci U.S.A. (2016) 113(26):7160-5. doi: 10.1073/pnas.1606272113

43. Schlipp A, Schinner C, Spindler V, Vielmuth F, Gehmlich K, Syrris P, et al. Desmoglein-2 interaction is crucial for cardiomyocyte cohesion and function. Cardiovasc Res (2014) 104(2):245-57. doi: 10.1093/cvr/cvu206

44. Saito M, Tucker DK, Kohlhorst D, Niessen CM, Kowalczyk AP. Classical and desmosomal cadherins at a glance. J Cell Sci (2012) 125(Pt 11):2547-52. doi: $10.1242 /$ jcs.066654

45. Niessen CM, Leckband D, Yap AS. Tissue organization by cadherin adhesion molecules: dynamic molecular and cellular mechanisms of morphogenetic regulation. Physiol Rev (2011) 91(2):691-731. doi: 10.1152/physrev.00004.2010

46. Bell G. Models for the specific adhesion of cells to cells. Science (1978) 200 (4342):618-27. doi: 10.1126/science.347575

47. Wieland JA, Gewirth AA, Leckband DE. Single Molecule Adhesion Measurements Reveal Two Homophilic Neural Cell Adhesion Molecule Bonds with Mechanically Distinct Properties. J Biol Chem (2005) 280 (49):41037-46. doi: 10.1074/jbc.M503975200
48. Tsunoda K, Ota T, Aoki M, Yamada T, Nagai T, Nakagawa T, et al. Induction of pemphigus phenotype by a mouse monoclonal antibody against the amino-terminal adhesive interface of desmoglein 3. J Immunol (2003) 170(4):2170-8. doi: 10.4049/jimmunol.170.4.2170

49. Nie Z, Merritt A, Rouhi-Parkouhi M, Tabernero L, Garrod D. Membraneimpermeable cross-linking provides evidence for homophilic, isoform-specific binding of desmosomal cadherins in epithelial cells. J Biol Chem (2011) 286 (3):2143-54. doi: 10.1074/jbc.M110.192245

50. Lowndes M, Rakshit S, Shafraz O, Borghi N, Harmon RM, Green KJ, et al. Different roles of cadherins in the assembly and structural integrity of the desmosome complex. J Cell Sci (2014) 127(Pt 10):2339-50. doi: 10.1242/ jcs. 146316

51. Ungewiss H, Rotzer V, Meir M, Fey C, Diefenbacher M, Schlegel N, et al. Dsg2 via Src-mediated transactivation shapes EGFR signaling towards cell adhesion. Cell Mol Life Sci (2018) 75(22):4251-68. doi: 10.1007/s00018-0182869-x

52. Daday C, Kolsek K, Grater F. The mechano-sensing role of the unique SH3 insertion in plakin domains revealed by Molecular Dynamics simulations. Sci Rep (2017) 7(1):11669. doi: 10.1038/s41598-017-11017-2

53. Buckley CD, Tan J, Anderson KL, Hanein D, Volkmann N, Weis WI, et al. Cell adhesion. The minimal cadherin-catenin complex binds to actin filaments under force. Science (2014) 346(6209):1254211. doi: 10.1126/ science. 1254211

54. Schinner C, Vielmuth F, Rotzer V, Hiermaier M, Radeva MY, Co TK, et al. Adrenergic Signaling Strengthens Cardiac Myocyte Cohesion. Circ Res (2017) 120 (8):1305-17. doi: 10.1161/CIRCRESAHA.116.309631

55. Rakshit S, Zhang Y, Manibog K, Shafraz O, Sivasankar S. Ideal, catch, and slip bonds in cadherin adhesion. Proc Natl Acad Sci U.S.A. (2012) 109(46):1881520. doi: 10.1073/pnas.1208349109

56. Baumgartner W, Hinterdorfer P, Ness W, Raab A, Vestweber D, Schindler H, et al. Cadherin interaction probed by atomic force microscopy. Proc Natl Acad Sci U.S.A. (2000) 97(8):4005-10. doi: 10.1073/pnas.070052697

57. Zhu C, McEver RP. Catch bonds: physical models and biological functions. Mol Cell Biomech (2005) 2(3):91-104.

58. Spindler V, Vielmuth F, Schmidt E, Rubenstein DS, Waschke J. Protective endogenous cyclic adenosine 5'-monophosphate signaling triggered by pemphigus autoantibodies. J Immunol (2010) 185(11):6831-8. doi: 10.4049/ jimmunol.1002675

59. Brennan D, Hu Y, Medhat W, Dowling A, Mahoney MG. Superficial dsg2 expression limits epidermal blister formation mediated by pemphigus foliaceus antibodies and exfoliative toxins. Dermatol Res Pract (2010) 2010:410278. doi: 10.1155/2010/410278

Conflict of Interest: The authors declare that the research was conducted in the absence of any commercial or financial relationships that could be construed as a potential conflict of interest.

Copyright (c) 2020 Sigmund, Steinert, Egu, Bayerbach, Waschke and Vielmuth. This is an open-access article distributed under the terms of the Creative Commons Attribution License (CC BY). The use, distribution or reproduction in other forums is permitted, provided the original author(s) and the copyright owner(s) are credited and that the original publication in this journal is cited, in accordance with accepted academic practice. No use, distribution or reproduction is permitted which does not comply with these terms. 